\title{
On Termination of 4-fold Semi-stable Log Flips
}

By

\author{
Osamu FujINO*
}

\begin{abstract}
In this paper, we prove the termination of 4-fold semi-stable log flips under the assumption that there always exist 4-fold (semi-stable) log flips.
\end{abstract}

\section{$\S 1$. Introduction}

One of the most important conjectures in the (log) minimal model program ((log) MMP, for short) is (log) Flip Conjecture II. It claims that any sequence of (log) flips:

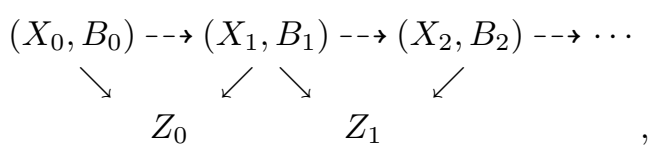

has to terminate after finitely many steps. In the non-log case, the conjecture in dimension 4 was proved for the terminal flips by Kawamata in [KMM], and for the terminal flops by Matsuki in [M1]. For the log case, we proved it for 4fold canonical flips in [F2], which is a first step to prove the log Flip Conjecture II in dimension 4 . We note that the main theorem of [F2] contains the above mentioned results of Kawamata and Matsuki. See also [F3].

Recently, Shokurov treats the log Flip Conjecture II in a much more general setting. For the details, see [S2] and [S3].

The main purpose of this paper is to prove the following theorem, which is a 4-dimensional analogue of [KM, Theorem 7.7], under the assumption that there always exist 4-fold (semi-stable) log flips (see Assumption 1.1 below). We

Communicated by S. Mori. Received August 22, 2003.

2000 Mathematics Subject Classification(s): Primary 14E30; Secondary 14J35, 14E05.

*Graduate School of Mathematics, Nagoya University, Chikusa-ku Nagoya 464-8602, Japan.

(C) 2005 Research Institute for Mathematical Sciences, Kyoto University. All rights reserved. 
will prove it by the crepant descent technique by Kawamata and Kollár (see [Ka1], [Ka3], [Ko], and $\left[\mathrm{K}^{+}\right.$, Chapter 6]). For the details of the (log) semi-stable MMP, see [KM, §7.1]. Roughly speaking, $(n+1)$-dimensional log semi-stable MMP is a kind of $n$-dimensional $\log$ MMP in families. So, it will play important roles in the study of the moduli of $n$-dimensional varieties.

We will work over $\mathbb{C}$, the complex number field, throughout this paper.

Theorem 1.1 (Termination of 4-fold semi-stable log flips). Let $(X, B)$ be a $\mathbb{Q}$-factorial projective 4-dimensional dlt pair, $\mu: X \longrightarrow Y$ a projective surjective morphism and $\nu: Y \longrightarrow C$ a flat morphism to a non-singular curve $C$ such that $f:=\nu \circ \mu:(X, B) \longrightarrow C$ is a dlt morphism (for the definition of dlt morphisms, see Definition 2.2 below). Then an arbitrary sequence of extremal $\left(K_{X}+B\right)$-flips over $Y$ is finite.

In the proof of Theorem 1.1, we need the following assumption: Assumption 1.1.

Assumption 1.1. Let $(X, B)$ be a 4-dimensional klt pair and $f: X \longrightarrow$ $Z$ a flipping contraction with respect to $K_{X}+B$. Then $f$ has a flip.

We note that all the flips we need here are 4-fold semi-stable (log) flips, which are special ones of klt flips in Assumption 1.1 (see Definition 2.3 and $\S 5$ Appendix). In Section 5, we will slightly generalize Theorem 1.1. We omit the details here since it is technical. Recently, Shokurov announced a proof of the existence of 4-fold log flips in [S1]. So, this assumption seems to be reasonable. We recommend the readers to see $[\mathrm{S} 1]$.

For the proof of Theorem 1.1, we need the following two theorems. First, we recall the special termination theorem. For the details, see [S1, Section 2] and $[\mathrm{F} 1]$.

Theorem 1.2 (4-dimensional special termination). $\quad$ Let $(X, B)$ be $a \mathbb{Q}$ factorial dlt 4-fold. Consider a sequence of extremal $\left(K_{X_{i}}+B_{i}\right)$-flips starting from $(X, B)=\left(X_{0}, B_{0}\right)$ :

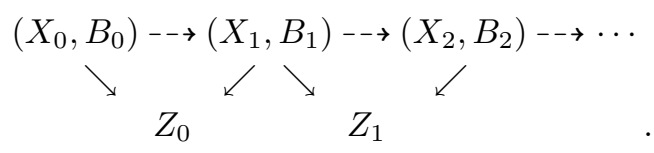

Then after finitely many flips, flipping locus (and thus the flipped locus) is disjoint from $\left\llcorner B_{i}\right\lrcorner$. 
Next, the following theorem is contained in [F2]. See also $[F 3, \S 5]$.

Theorem 1.3 (Termination of 4-fold terminal flips). Let $X$ be a normal projective 4-fold and $B$ an effective $\mathbb{Q}$-divisor such that $(X, B)$ is terminal, that is, $\operatorname{discrep}(X, B)>0$. Consider a sequence of $\left(K_{X_{i}}+B_{i}\right)$-flips starting from $(X, B)=\left(X_{0}, B_{0}\right)$ :

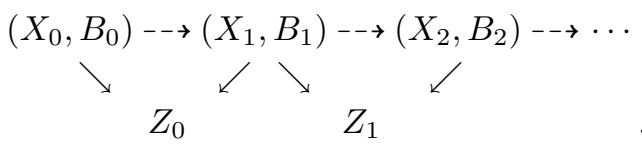

Then this sequence terminates after finitely many steps.

We note that we do not need Assumption 1.1 in the proofs of Theorems 1.2 and 1.3 .

We summarize the contents of this paper: In Section 2, we recall some basic definitions and introduce a new notion: plt morphism. Section 3 is the preparation for the main theorem. We define a couple of invariants for plt morphisms. Section 4 is devoted to the proof of the main theorem: Theorem 1.1. Finally, Section 5 is an appendix, where we slightly generalize Theorem 1.1.

Notation. Let $\mathbb{Z}_{>0}$ (resp. $\left.\mathbb{Z}_{\geq 0}\right)$ be a set of positive (resp. non-negative) integers. For $d \in \mathbb{Q}$, let $\llcorner d\lrcorner=\max \{t \in \mathbb{Z} \mid t \leq d\}$ and $\{d\}=d-\llcorner d\lrcorner$. Let $D=\sum d_{i} D_{i}$ be a $\mathbb{Q}$-divisor such that all the $D_{i}$ 's are distinct prime divisors. We put $\llcorner D\lrcorner=\sum\left\llcorner d_{i}\right\lrcorner D_{i}$ (the round down of $D$ ) and $\{D\}=\sum\left\{d_{i}\right\} D_{i}$ (the fractional part of $D)$.

\section{$\S 2 . \quad$ Preliminaries}

In this section, we collect basic properties and definitions.

2.1. First, let us recall the definitions of discrepancies and singularities of pairs.

Definition 2.1 (Discrepancies and singularities for pairs). Let $X$ be a normal variety and $D=\sum d_{i} D_{i}$ a $\mathbb{Q}$-divisor on $X$, where $D_{i}$ is irreducible for every $i$ and $D_{i} \neq D_{j}$ for $i \neq j$, such that $K_{X}+D$ is $\mathbb{Q}$-Cartier. Let $f: Y \longrightarrow X$ be a proper birational morphism from a normal variety $Y$. Then we can write

$$
K_{Y}=f^{*}\left(K_{X}+D\right)+\sum a(E, X, D) E,
$$


where the sum runs over all the distinct prime divisors $E \subset Y$, and $a(E, X, D)$ $\in \mathbb{Q}$. This $a(E, X, D)$ is called the discrepancy of $E$ with respect to $(X, D)$. We define

$$
\operatorname{discrep}(X, D):=\inf _{E}\{a(E, X, D) \mid E \text { is exceptional over } X\} \text {. }
$$

From now on, we assume that $0 \leq d_{i} \leq 1$ for every $i$. We say that $(X, D)$ is

$$
\left\{\begin{array}{l}
\text { terminal } \\
\text { canonical } \\
\text { klt } \\
\text { plt } \\
\text { lc }
\end{array}\right.
$$

Here klt is short for Kawamata log terminal, plt for purely log terminal, and lc for log canonical.

If there exists a $\log$ resolution $f: Y \longrightarrow X$ of $(X, D)$, that is, $Y$ is nonsingular, the exceptional locus $\operatorname{Exc}(f)$ is a divisor, and $\operatorname{Exc}(f) \cup f^{-1}(\operatorname{Supp} D)$ is a simple normal crossing divisor, such that $a\left(E_{i}, X, D\right)>-1$ for every exceptional divisor $E_{i}$ on $Y$, then the pair $(X, D)$ is called $d l t$. Here, dlt is short for divisorial log terminal.

2.2. Next, let us recall the definition of dlt morphisms and define plt morphisms.

Definition 2.2 ([KM, Definition 7.1]). $\quad$ Let $X$ be a normal variety, $B$ an effective $\mathbb{Q}$-divisor on $X$ and $f: X \longrightarrow C$ a non-constant morphism to a non-singular curve $C$. We say that $f:(X, B) \longrightarrow C$ is dlt (resp. plt) if $\left(X, B+f^{*} P\right)$ is dlt (resp. plt) for every closed point $P \in C$. We note that if $(X, B) \longrightarrow C$ is plt, then $(X, B)$ is klt.

The following lemma is a variant of adjunction and the inversion of adjunction. For the proof, see [KM, Theorem 5.50 (1), Proposition 5.51].

Lemma 2.1. Let $(X, B)$ be a klt pair and $f:(X, B) \longrightarrow C$ a dlt morphism. Then the following four conditions are equivalent.

(1) $f:(X, B) \longrightarrow C$ is a plt morphism.

(2) every connected component of any fiber is irreducible.

(3) $\left(F,\left.B\right|_{F}\right)$ is a klt pair for any fiber $F$. 
(4) all the fibers of $f$ are normal.

The next lemma is an analogue of [KM, Lemma $7.2(4)]$. It easily follows from the definition of dlt pairs (see [KM, Definition 2.37]). We leave the details to the readers.

Lemma 2.2. $\quad$ Let $(X, B)$ be a klt pair and $f:(X, B) \longrightarrow C$ a dlt morphism. If $E$ is an exceptional divisor over $X$ such that the center of $E$ on $X$ is contained in a fiber, then the discrepancy $a(E, X, B)>0$.

We note the following properties, which is an easy consequence of the negativity lemma (cf. [KM, Lemma 3.38]).

Lemma 2.3 (cf. [KM, Corollary 3.44]). Let $\phi:(X, B) \rightarrow\left(X^{+}, B^{+}\right)$ be either $a\left(K_{X}+B\right)$-flip over $Y$ or a divisorial contraction of a $\left(K_{X}+B\right)$ negative extremal ray over $Y, f: Y \longrightarrow C$ a flat morphism onto a non-singular curve $C$, and $h:=f \circ g:(X, B) \longrightarrow C$ a dlt (resp. plt) morphism. Then $h^{+}:\left(X^{+}, B^{+}\right) \longrightarrow C$ is also a dlt (resp. plt) morphism.

2.3. Finally, we define semi-stable log flips (cf. [KM, Theorem 7.8]).

Definition 2.3. $\quad$ Let $(X, B)$ be a dlt pair and $f: X \longrightarrow W$ a flipping contraction with respect to $K_{X}+B$, that is, $f$ is small and $-\left(K_{X}+B\right)$ is $f$-ample. We assume that $f$ is extremal, where "extremal" means that $X$ is $\mathbb{Q}$-factorial and the relative Picard number $\rho(X / W)=1$. Assume that there exists a flat morphism $g: W \longrightarrow C$ to a smooth curve such that $h:=g \circ f$ is dlt. Then the flip $f^{+}: X^{+} \longrightarrow W$ of $f$ :

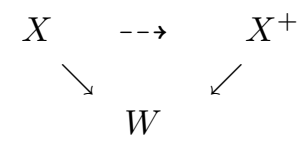

that is,

(i) $f^{+}$is small,

(ii) $K_{X^{+}}+B^{+}$is $f^{+}$-ample, where $B^{+}$is the strict transform of $B$,

is called a semi-stable $(\log )$ flip of $f$. Furthermore, if $(X, B)$ is terminal, that is, $\operatorname{discrep}(X, B)>0$, then we call $f^{+}$a semi-stable terminal flip of $f$.

We treat only two examples here. 
Example 1 (4-fold semi-stable flip). $\quad$ Let $V$ be a projective 3 -fold with $\mathbb{Q}$-factorial terminal singularities and

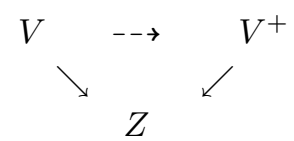

an extremal $K_{V^{-}}$flip. We define $X:=V \times \mathbb{P}^{1}, X^{+}:=V^{+} \times \mathbb{P}^{1}$, and $W:=Z \times \mathbb{P}^{1}$. We put $Y:=C:=\mathbb{P}^{1}$. Then

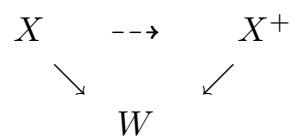

is an extremal 4-fold semi-stable flip over $Y$. We note that the second projection $X \longrightarrow C$ is a plt morphism. It is not difficult to see that $\rho(X / W)=1$ and $X$ is $\mathbb{Q}$-factorial. In this case, the flipping and flipped loci are dominant onto $C$.

The following example is a 4-fold toric flip. We quote it from [M2, ExampleClaim 14-2-8].

Example 2 (Toric 4-dimensional flip). $\quad$ Let $N_{1}=\mathbb{Z}^{4}$ and $N_{2}=\mathbb{Z}$. We put

$$
\begin{aligned}
& v_{1}=(1,0,0,0) \\
& v_{2}=(0,1,0,0) \\
& v_{3}=(0,0,1,0) \\
& v_{4}=(0,0,0,1) \\
& v_{5}=(1,1,-1,-1)
\end{aligned}
$$

and consider the following two cones,

$$
\tau_{5}=\left\langle v_{1}, v_{2}, v_{3}, v_{4}\right\rangle, \quad \tau_{4}=\left\langle v_{1}, v_{2}, v_{3}, v_{5}\right\rangle
$$

We define the two fans,

$$
\begin{aligned}
\Delta & =\left\{\tau_{4}, \tau_{5}, \text { and their faces }\right\}, \\
\Delta^{\prime} & =\left\{\left\langle v_{1}, v_{2}, v_{3}, v_{4}, v_{5}\right\rangle, \text { and its faces }\right\} .
\end{aligned}
$$

We consider the toric morphism $g: X(\Delta) \longrightarrow X\left(\Delta^{\prime}\right)$. This is a flipping contraction. We consider the first projection $p: N_{1} \longrightarrow N_{2}$. This $p$ induces 
$f: X\left(\Delta^{\prime}\right) \longrightarrow \mathbb{A}^{1}=X(\langle e\rangle)$, where $e=1 \in N_{2}$. By this morphism, $X(\Delta) \longrightarrow$ $X\left(\Delta^{\prime}\right)$ is a semi-stable flipping contraction. We can construct the flip of $g$ and determine the exceptional locus of $g$ and so on. Note that $\operatorname{Exc}(g)=V\left(\left\langle v_{1}, v_{2}\right\rangle\right)$ and $(f \circ g)^{-1}(0)=V\left(\left\langle v_{1}\right\rangle\right) \cup V\left(\left\langle v_{5}\right\rangle\right)$. So, this flip is of type (B) in [Kc, Main Theorem 0.5]. For the details, see [M2, Example-Claim 14-2-8, Remark 14-2-9].

The related topics of Example 2 are [Ka2], [T1], and [T2].

\section{§3. Preparation}

In this section, we make preparations for the proof of the main theorem: Theorem 1.1.

3.1. We write a sequence of 4-fold semi-stable flips over $Y$ as follows:

$$
(X, B)=:\left(X_{0}, B_{0}\right) \rightarrow\left(X_{1}, B_{1}\right) \rightarrow\left(X_{2}, B_{2}\right)-\rightarrow \cdots
$$

where $\phi_{i}: X_{i} \longrightarrow W_{i}$ is an extremal flipping contraction with respect to $K_{X_{i}}+B_{i}$ over $Y$ and $\phi_{i}^{+}: X_{i+1} \longrightarrow W_{i}$ is the flip of $\phi_{i}$ for every $i$.

By the special termination theorem: Theorem 1.2, all the flipping and flipped loci are disjoint from $\left\llcorner B_{i}\right\lrcorner$ after finitely many flips. Therefore, we can assume that all the flipping and flipped loci are disjoint from $\left\llcorner B_{i}\right\lrcorner$ for every $i$ by shifting the index $i$. So, we can replace $B_{i}$ with its fractional part $\left\{B_{i}\right\}$ and assume that $\left(X_{i}, B_{i}\right)$ is klt. From now on, we assume that $\left(X_{i}, B_{i}\right)$ is klt for every $i$.

Let us recall the following definition.

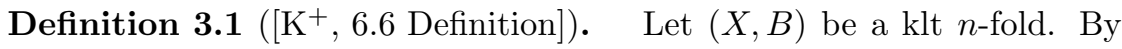
[KM, Proposition 2.3.6], there are only finitely many exceptional divisors with non-positive discrepancies. The number of these divisors is denoted by $e(X, B)$. Thus $(X, B)$ is terminal if and only if $e(X, B)=0$ by the definition of terminal pairs.

3.2. We prove Theorem 1.1 by induction on $e(X, B)$.

If $e(X, B)=0$, then $(X, B)$ is terminal. Thus a sequence of flips always terminates by Theorem 1.3. Therefore, we assume that the theorem holds for $e(X, B) \leq e-1$, and prove it in case $e(X, B)=e$. We note that $e\left(X_{i}, B_{i}\right) \geq$ $e\left(X_{i+1}, B_{i+1}\right)$ for all $i$ by the negativity lemma (cf. [KM, Lemma 3.38]). 
3.3. First, we add $f^{*} P$ to $B$, where $P$ is a closed point of $C$. We may regard the $\left(K_{X}+B\right)$-flips as the $\left(K_{X}+B+f^{*} P\right)$-flip. Then by Theorem 1.2, we can assume that all the flipping and flipped loci are not dominant onto $C$ after finitely many flips. Thus, by shifting the index $i$ we can assume that all the flipping and flipped loci are contained in some fibers.

So, we can assume that there are no semi-stable flips like Example 1.

3.4. $\quad$ Next, we add $\sum_{P} f^{*} P$ to $B$, where $P$ runs through all the closed points of $C$ such that $f^{*} P$ is not normal. By Theorem 1.2 again, we can assume that all the flipping and flipped loci are disjoint from non-normal fibers. We note that the normality of fibers are preserved by flips (see Lemmas 2.1 and 2.3). Therefore, we can assume that there exists a non-empty Zariski open set $U$ of $C$ such that all the flips occur over this open set $U$ and $\left(X_{i}, B_{i}\right) \longrightarrow C$ is a plt morphism over $U$ (see Definition 2.2).

We recall the definition of $r(X, B)$.

Definition 3.2 $\left(\left[\mathrm{K}^{+}, 6.9 .8\right.\right.$ Definition $\left.]\right) . \quad$ Let $(X, B)$ be a klt $n$-fold. We put

$$
s(X, B):=\min \{a(E, X, B)>0 \mid E \text { is exceptional over } X\} .
$$

Then we define

$$
r(X, B):=\left(4\left\ulcorner s(X, B)^{-1}\right\urcorner\right) ! \in \mathbb{Z}_{>0} .
$$

We generalize the invariants $e(X, B), r(X, B)$, and $\operatorname{discrep}(X, B)$ for plt morphisms. By Lemma 2.1 (3), a plt morphism is a family of klt pairs. So, the following definition is natural.

Lemma-Definition 3.1. Let $f:(X, B) \longrightarrow C$ be a plt morphism. Then

$$
\begin{gathered}
0 \leq \max _{F} e\left(F,\left.B\right|_{F}\right)<\infty, \\
\max _{F} r\left(F,\left.B\right|_{F}\right) \in \mathbb{Z}_{>0}, \quad \text { and } \\
-1<\min _{F} \operatorname{discrep}\left(F,\left.B\right|_{F}\right) \leq 1,
\end{gathered}
$$

where $F$ runs through all the fibers of $f$. We define

$$
\begin{gathered}
e(f ;(X, B)):=\max _{F} e\left(F,\left.B\right|_{F}\right), \\
r(f ;(X, B)):=\max _{F} r\left(F,\left.B\right|_{F}\right), \quad \text { and } \\
\operatorname{discrep}(f ;(X, B)):=\min _{F} \operatorname{discrep}\left(F,\left.B\right|_{F}\right) .
\end{gathered}
$$


Proof. We note that $K_{F}+\left.B\right|_{F}:=\left.\left(K_{X}+B+F\right)\right|_{F}$ is klt by adjunction (see Lemma 2.1). Take a log resolution $g: Z \longrightarrow X$ of the pair $(X, B)$ as in [KM, Proposition $2.36(1)]$. We write

$$
K_{Z}+D-E=g^{*}\left(K_{X}+B\right),
$$

where $D=\sum a_{i} D_{i}$ and $E=\sum b_{j} E_{j}$ are both effective and have no common irreducible component. Let $G=\sum G_{k}$ be the $g$-exceptional divisor such that $a\left(G_{k}, X, B\right)=0$ for every $k$. We can assume that $\operatorname{Supp}(D \cup G)$ is non-singular. There exists a non-empty Zariski open set $U \subset C$ such that $f \circ g$ is smooth and $\operatorname{Supp}(D \cup E \cup G)$ is relatively normal crossing over $U$. We can assume that $g\left(D_{i}\right) \longrightarrow C, g\left(E_{j}\right) \longrightarrow C$, and $g\left(G_{k}\right) \longrightarrow C$ are flat over $U$ for every $i, j$, and $k$ after shrinking $U$. Over this open set $U, e\left(F,\left.B\right|_{F}\right), r\left(F,\left.B\right|_{F}\right)$ (more precisely, $s\left(F,\left.B\right|_{F}\right)$ ), and $\operatorname{discrep}\left(F,\left.B\right|_{F}\right)$ are constant. Thus, we can check the claim. Therefore, $e(f ;(X, B)), r(f ;(X, B))$, and $\operatorname{discrep}(f ;(X, B))$ are well-defined.

The next proposition will play crucial roles in the proof of the main theorem.

Proposition 3.1. Let $f:(X, B) \longrightarrow C$ be a plt morphism and $D$ a $\mathbb{Q}$-Cartier Weil divisor on $X$. Then $m D$ is Cartier if and only if so is $\left.m D\right|_{F}$ for every fiber $F$. In particular, if $K_{X}$ is $\mathbb{Q}$-Cartier, then $m K_{X}$ is Cartier if and only if so is $m K_{F}$ for every fiber $F$.

Proof. See, for example, [HL, Lemma 2.1.7]. We note that $(X, B+F)$ is plt and $F$ is Cartier. Thus, in a neighborhood of $F, \operatorname{codim}_{X}(\operatorname{Sing} X \cap F) \geq 3$, where $\operatorname{Sing} X$ is the singular locus of $X$. So, $\left.\mathcal{O}_{X}(m D)\right|_{F} \simeq \mathcal{O}_{F}\left(\left.m D\right|_{F}\right)$ and $\left.\mathcal{O}_{X}\left(m\left(K_{X}+F\right)\right)\right|_{F} \simeq \mathcal{O}_{F}\left(m K_{F}\right)$ for every $m \in \mathbb{Z}_{\geq 0}$ (cf. [KM, Proposition $5.26])$.

We recall the result in $\left[\mathrm{K}^{+}, 6.11\right.$ Theorem]. For the proof, see $\left[\mathrm{K}^{+}\right.$, $(6.11 .5)]$.

Theorem 3.1. $\quad$ Let $(V, \Delta)$ be a klt 3-fold and $E$ a $\mathbb{Q}$-Cartier Weil divisor on $V$. Then $m E$ is Cartier for some

$$
1 \leq m \leq r(V, \Delta)^{2^{e(V, \Delta)}}\left(\frac{3}{1+\operatorname{discrep}(V, \Delta)}\right)^{2^{e(V, \Delta)}-1} .
$$


Theorem 3.2. $\quad$ Let $(X, B)$ be a klt 4-fold and $f:(X, B) \longrightarrow C$ a plt morphism. Let $E$ be a $\mathbb{Q}$-Cartier Weil divisor on $X$. Then $M E$ is Cartier for

$$
M:=M(f ;(X, B)):=(\ulcorner\varphi(f ;(X, B))\urcorner) ! \in \mathbb{Z}_{>0},
$$

where

$$
\varphi(f ;(X, B)):=r(f ;(X, B))^{2^{e(f ;(X, B))}}\left(\frac{3}{1+\operatorname{discrep}(f ;(X, B))}\right)^{2^{e(f ;(X, B))}-1} .
$$

Let $U$ be a non-empty Zariski open subset of $C$. Then the restriction

$$
\left.f\right|_{f^{-1}(U)}:\left.(X, B)\right|_{f^{-1}(U)} \longrightarrow U
$$

is a plt morphism and $M\left(\left.f\right|_{f^{-1}(U)} ;\left.(X, B)\right|_{f^{-1}(U)}\right)$ divides $M(f ;(X, B))$.

Proof. It is obvious by Theorem 3.1. We note that if $E$ is not dominant onto $C$, then $E$ is Cartier (see Lemma 2.1). The latter statement directly follows from the definition of $M$.

\section{$\S 4 . \quad$ Proof of the Main Theorem}

We go back to the proof of the main theorem: Theorem 1.1. Our proof is similar to that of $\left[\mathrm{K}^{+}, 6.11\right.$ Theorem].

Proof of Theorem 1.1. We start the proof of the main theorem.

Step 1. First, we take a log resolution of $(X, B)$. We write $p: Z \longrightarrow X$ and

$$
K_{Z}+p_{*}^{-1} B=p^{*}\left(K_{X}+B\right)+E-F,
$$

where $E$ and

$$
F:=\sum_{a_{i} \geq 0} a_{i} F_{i}
$$

are effective exceptional divisors and have no common irreducible components. If necessary, we further blow up $Z$. Then we can assume that $\sum_{a_{i} \geq 0} F_{i}$ contains all the exceptional divisors whose discrepancies are non-positive, $\operatorname{Supp}\left(p_{*}^{-1} B \cup\right.$ $\left.\sum F_{i}\right)$ is smooth and $\operatorname{Supp}\left(p_{*}^{-1} B \cup \sum F_{i} \cup(f \circ p)^{*} P\right)$ is simple normal crossing for every $P \in C$. We note that $F_{i}$ is dominant onto $C$ for every $i$ by Lemma 2.2 . By 3.2 , we can assume that $\sum F_{i} \neq 0$, that is, $e=e(X, B)>0$. We consider

$$
f \circ p:\left(Z, D^{\varepsilon}\right):=\left(Z, p_{*}^{-1} B+F+\varepsilon \sum_{i \neq 0} F_{i}\right) \longrightarrow C .
$$


It is easy to check that $\left(Z, D^{\varepsilon}\right)$ is terminal and $f \circ p:\left(Z, D^{\varepsilon}\right) \longrightarrow C$ is a dlt morphism for $0<\varepsilon \ll 1$. Run the $\log$ MMP over $X$. Then we obtain a sequence of flips and divisorial contractions over $X$ :

$$
\left(Z, D^{\varepsilon}\right):=\left(Z_{0}, D_{0}^{\varepsilon}\right) \rightarrow\left(Z_{1}, D_{1}^{\varepsilon}\right) \rightarrow \cdots \cdots\left(Z_{k}, D_{k}^{\varepsilon}\right)-\rightarrow \cdots .
$$

By Assumption 1.1, flips exist and by induction, any sequence of flips terminates since $e\left(Z_{k}, D_{k}^{\varepsilon}\right)<e=e(X, B)$ for every $k$ (see Remark below). Note that each flip in the above process is a semi-stable log flip. Then we obtain a relative log minimal model $q:\left(Z^{\prime}, B^{\prime}\right) \longrightarrow X$, which satisfies the following conditions:

(1) $f \circ q:\left(Z^{\prime}, B^{\prime}\right) \longrightarrow C$ is a dlt morphism.

(2) $f \circ q:\left(Z^{\prime}, B^{\prime}\right) \longrightarrow C$ is a plt morphism over $U$ (see 3.4).

(3) $e\left(Z^{\prime}, B^{\prime}\right)=e(X, B)-1$.

(4) $\left(Z^{\prime}, B^{\prime}\right)$ is a $\mathbb{Q}$-factorial klt pair.

(5) $K_{Z^{\prime}}+B^{\prime}=q^{*}\left(K_{X}+B\right)$, that is, $q$ is a log crepant morphism.

(6) the relative Picard numbers $\rho\left(Z^{\prime} / X\right)=1$ and $\rho\left(Z^{\prime} / W_{0}\right)=2$.

We note that $\alpha: Z \rightarrow Z^{\prime}$ is an isomorphism at the generic point of $F_{0}$ and contracts $E+\sum_{i \neq 0} F_{i}$.

Step 2. We put $p_{0}:\left(Z_{0}^{0}, B_{0}^{0}\right):=\left(Z^{\prime}, B^{\prime}\right) \longrightarrow X=: X_{0}$. We construct a sequence of flips $\rightarrow Z_{i}^{j} \rightarrow Z_{i}^{j+1} \rightarrow$ over $X_{i} \rightarrow X_{i+1}$ for every $i$. We assume that we already have $p_{i}:\left(Z_{i}^{0}, B_{i}^{0}\right) \longrightarrow X_{i}$. Run the $\log$ MMP to $\left(Z_{i}^{0}, B_{i}^{0}\right)$ over $W_{i}$. We obtain a sequence of flips and divisorial contractions over $W_{i}$ :

$$
Z_{i}^{0} \rightarrow Z_{i}^{1} \rightarrow \rightarrow \cdots \rightarrow Z_{i}^{k_{i}},
$$

and a $\log$ minimal model $\left(Z_{i}^{k_{i}}, B_{i}^{k_{i}}\right)$ for $\left(Z_{i}^{0}, B_{i}^{0}\right)$ over $W_{i}$. This is a so-called 2 ray games. Note that each flip in the above process is a semi-stable log flip. Since $\left(X_{i+1}, B_{i+1}\right)$ is the $\log$ canonical model of $\left(Z_{i}^{0}, B_{i}^{0}\right)$ over $W_{i}$, there exists a morphism $q_{i}: Z_{i}^{k_{i}} \longrightarrow X_{i+1}$.

Case A. If all the steps in the above log MMP are flips, then we have $K_{Z_{i}^{k_{i}}}+B_{i}^{k_{i}}=q_{i}^{*}\left(K_{X_{i+1}}+B_{i+1}\right)$. We define $p_{i+1}:\left(Z_{i+1}^{0}, B_{i+1}^{0}\right):=\left(Z_{i}^{k_{i}}, B_{i}^{k_{i}}\right)$ $\stackrel{Z_{i+1}}{\longrightarrow} X_{i+\text { We put }} c_{i+1}=0$ in this case.

Case B. If a divisorial contraction occurs in the above log MMP, then it is not difficult to see that the final step $\beta: Z_{i}^{k_{i}-1} \rightarrow Z_{i}^{k_{i}}$ is a divisorial 
contraction and $q_{i}: Z_{i}^{k_{i}} \longrightarrow X_{i+1}$ is an isomorphism (cf. [KM, Lemma 6.39] and $\left[\mathrm{K}^{+}, 6.5 .5\right.$ Proposition]). We note that other steps in the above log MMP are all flips. We also note that

$$
K_{Z_{i}^{k_{i}-1}}+B_{i}^{k_{i}-1}=\left(q_{i} \circ \beta\right)^{*}\left(K_{X_{i+1}}+B_{i+1}\right)+c_{i+1} F_{0}
$$

for $c_{i+1}>0$, where $F_{0}$ is the proper transform of $F_{0}$ on $Z_{i}^{k_{i}-1}$. Then we put $p_{i+1}:\left(Z_{i+1}^{0}, B_{i+1}^{0}\right):=\left(Z_{i}^{k_{i}-1}, B_{i}^{k_{i}-1}-c_{i+1} F_{0}\right) \longrightarrow X_{i+1}$.

Note that $\left(Z_{i+1}^{0}, B_{i+1}^{0}\right) \longrightarrow C$ is a dlt morphism. So, $\left(Z_{i}^{j}, B_{i}^{j}\right) \longrightarrow C$ is dlt for every $i, j$.

Step 3. We assume that the sequence

$$
X_{0} \rightarrow X_{1-\rightarrow} X_{2} \rightarrow \cdots \cdots-\rightarrow X_{i}-\rightarrow
$$

is infinite. If Case B occurs only finitely many times, then we can assume that all the steps are Case A. Then we obtain an infinite sequence of flips with respect to $K_{Z_{i}^{j}}+B_{i}^{j}$. Since $e\left(Z_{i}^{j}, B_{i}^{j}\right)<e(X, B)$, it is impossible. So, Case B occurs infinitely many times. The coefficient of $F_{0}$ in $B_{i+1}^{0}$, where $F_{0}$ is the proper transform of $F_{0}$ on $Z_{i+1}^{0}$, is

$$
a_{0}-\sum_{0 \leq j \leq i} c_{j+1}
$$

where $a_{0}:=-a\left(F_{0}, X, B\right) \geq 0$, that is, $a\left(F_{0}, X, B\right) \leq 0$. Let $U_{i+1}$ be a nonempty Zariski open set of $U$ such that flips $\left(X_{j}, B_{j}\right) \rightarrow\left(X_{j+1}, B_{j+1}\right)$ occur over $U \backslash U_{i+1}$ for $0 \leq j \leq i$. We note that it is sufficient to consider the coefficient of $F_{0}$ over $U_{i+1}$ since $F_{0}$ is irreducible and dominant onto $C$. Let $N$ be a positive integer such that $N B_{0}$ is a Weil divisor. Then $N B_{i}$ is also a Weil divisor for every $i$. By Theorem 3.2, $M N\left(K_{X_{i}}+B_{i}\right)$ is a Cartier divisor over $U_{i}$ for every $i$, where $M:=M\left(\left.h\right|_{(h)^{-1}(U)} ;\left.(X, B)\right|_{(h)^{-1}(U)}\right)$. We note that $M\left(\left.h\right|_{(h)^{-1}\left(U_{i}\right)} ;\left.(X, B)\right|_{(h)^{-1}\left(U_{i}\right)}\right)$ divides $M$ by Lemma 2.3 and that $\left(X_{i}, B_{i}\right)$ is isomorphic to $(X, B)$ over $U_{i}$. Thus $M N B_{i}^{0}$ is a Weil divisor over $U_{i}$ for every $i$. So, we have that $M N c_{j} \in \mathbb{Z}_{\geq 0}$ for every $j$. Therefore, after finitely many steps, the coefficient of $F_{0}$ in $B_{i+1}^{0}$ is negative, that is, the discrepancy $a\left(F_{0}, X_{i+1}, B_{i+1}\right)>0$. Thus, $e\left(X_{i+1}, B_{i+1}\right)<e=e(X, B)$. So, a sequence of flips terminates by the induction on $e(X, B)$. This is a contradiction.

We complete the proof of Theorem 1.1.

Remark. Note that for the proof of the termination in case $e(X, B)=$ $e$, we use the existence and the termination of semi-stable log flips only for $e(*, *) \leq e-1$. 


\section{$\S 5 . \quad$ Appendix}

It is not difficult to see that the existence of 4-dimensional semi-stable terminal flips implies that of all the 4-dimensional semi-stable log flips. It is essentially proved in the proof of Theorem 1.1.

We assume the existence of 4-dimensional semi-stable terminal flips as follows.

Assumption 5.1. Let $(X, B)$ be a 4-dimensional terminal pair and $f$ : $X \longrightarrow Z$ an extremal flipping contraction with respect to $K_{X}+B$. Assume that there exists a flat morphism $g: Z \longrightarrow C$ to a smooth curve such that $h:=g \circ f$ is dlt. Then $f$ has a semi-stable terminal flip.

As mentioned above, Assumption 5.1 implies the existence of all the 4dimensional semi-stable log flips (cf. $\left[\mathrm{K}^{+}, 6.4,6.5,6.11\right.$ Theorem]).

Proposition 5.1. $\quad$ Let $(X, B)$ be a $\mathbb{Q}$-factorial projective 4-dimensional dlt pair and $f: X \longrightarrow W$ an extremal flipping contraction. Assume that there exists a flat morphism $g: W \longrightarrow C$ to a smooth curve such that $h:=g \circ f$ is dlt. Then Assumption 5.1 implies that the semi-stable log flip of $f$ exists.

Proof. We can assume that $(X, B)$ is klt by replacing $B$ with $(1-\varepsilon) B$ for $0<\varepsilon \ll 1$. If $e(X, B)=0$, then the flip exists by Assumption 5.1. Therefore, we assume that semi-stable log flips exist and any sequence of them terminates for $e(*, *) \leq e-1$, and prove the existence of the flip in case $e(X, B)=e$ (see also Remark in $\S 4)$. On this assumption, Step 1 in the proof of Theorem 1.1 works without any changes. So, we obtain $q: Z^{\prime} \longrightarrow X$ as in the proof of Theorem 1.1. Run the $\log$ MMP to $\left(Z^{\prime}, B^{\prime}\right)$ over $W$. We obtain a log minimal model $\left(Z^{\prime \prime}, B^{\prime \prime}\right)$ for $\left(Z^{\prime}, B^{\prime}\right)$ over $W$ (see Step 2 in the proof of Theorem 1.1). Note that if a divisorial contraction occurs, then it is the final step of the above log MMP (see Case B in Step 2), and any sequence of flips in this process terminates by the assumption. Since $\left(Z^{\prime \prime}, B^{\prime \prime}\right)$ is klt, we obtain the log canonical model $\left(X^{+}, B^{+}\right)$for $\left(Z^{\prime}, B^{\prime}\right)$ over $W$ by the relative base point free theorem. It is well-known that $\left(X^{+}, B^{+}\right)$is the required flip.

Remark. Note that for the proof of the existence of semi-stable log flips in case $e(X, B)=e$, we use the existence and the termination of semi-stable $\log$ flips only for $e(*, *) \leq e-1$.

So, by Proposition 5.1, we can generalize Theorem 1.1 slightly. Note Remarks in $\S 4$ and $\S 5$. 
Corollary 5.1. Assumption 5.1 implies Theorem 1.1 .

\section{Acknowledgments}

I would like to thank Professors V. V. Shokurov and Hiromichi Takagi for comments.

\section{References}

[F1] Fujino, O., On special termination and reduction theorem, to be contained in "Flips for 3-folds and 4-folds" by Corti, A., Ambro, F., Fujino, O., McKernan, J. and Takagi, H.

[F2] — Termination of 4-fold canonical flips, Publ. RIMS, Kyoto Univ., 40 (2004), 231-237.

[F3] Addendum to "Termination of 4-fold canonical flips" [Publ. RIMS, Kyoto Univ., 40 (2004), 231-237]; Publ. RIMS, Kyoto Univ., 41 (2005), 252-257.

[HL] Huybrechts, D. and Lehn, M., The geometry of moduli spaces of sheaves, Aspects of Mathematics, E31. Friedr. Vieweg \& Sohn, Braunschweig, 1997.

[Kc] Kachi, Y., Flips of semi-stable 4-folds whose degenerate fibers are unions of Cartier divisors which are terminal factorial 3-folds, Math. Ann., 307 (1997), 647-662.

[Ka1] Kawamata, Y., Crepant blowing-up of 3-dimensional canonical singularities and its application to degenerations of surfaces, Ann. of Math. (2), 127 (1988), 93-163.

[Ka2] Small contractions of four-dimensional algebraic manifolds, Math. Ann., 284 (1989), 595-600.

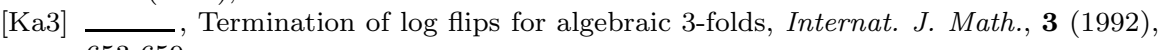
653-659.

[KMM] Kawamata, Y., Matsuda, K. and Matsuki, K., Introduction to the Minimal Model Problem, in Algebraic Geometry, Sendai 1985, Adv. Stud. Pure Math., 10 (1987), Kinokuniya and North-Holland, 283-360.

[Ko] Kollár, J., Flops, Nagoya Math. J., 113 (1989), 15-36.

[KM] Kollár, J. and Mori, S., Birational geometry of algebraic varieties, Cambridge Tracts in Mathematics, Vol. 134, 1998.

$\left[\mathrm{K}^{+}\right]$Kollár, J., et al., Flips and Abundance for algebraic threefolds, Astérisque, 211 (1992)

[M1] Matsuki, K., Termination of flops for 4-folds, Amer. J. Math., 113 (1991), 835-859.

[M2] Introduction to the Mori program, Universitext. Springer-Verlag, New York, 2002.

[S1] Shokurov, V. V., Prelimiting flips, In Birational Geometry: Linear systems and finitely generated algebras. A collection of papers. Iskovskikh, V. A. and Shokurov, V. V. Editors, Proc. of Steklov Institute, 240 (2003), 75-213.

[S2] L Letters of a bi-rationalist, IV, Geometry of log flips, Algebraic geometry, 313-328, de Gruyter, Berlin, 2002.

[S3] _ Letters of a bi-rationalist, V, Minimal log discrepancies and termination of log flips. (Russian) Tr. Mat. Inst. Steklova, 246 (2004), Algebr. Geom. Metody, Svyazi i Prilozh., 328-351.

[T1] Takagi, H., Remarks on Gorenstein terminal fourfold flips, J. Math. Sci. Univ. Tokyo, 5 (1998), 149-164.

[T2] _ The Existence of Gorenstein terminal fourfold flips, a revised version of [T1], Preprint 1999. 